\title{
Tentative Detection of the Rotation of Eris
}

\author{
Henry G. Roe ${ }^{\mathrm{a}}$, Rosemary E. Pike ${ }^{\mathrm{b}}$, Michael E. Brown ${ }^{\mathrm{c}}$

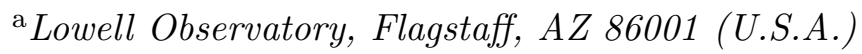 \\ ${ }^{\mathrm{b}}$ Gemini Observatory, Hilo, HI 96720 (U.S.A.) \\ ${ }^{\mathrm{c}}$ California Institute of Technology, Division of Geological and Planetary Sciences, \\ Pasadena, CA 91125 (U.S.A.)
}

\begin{abstract}
We report a multi-week sequence of B-band photometric measurements of the dwarf planet Eris using the Swift satellite. The use of an observatory in low-Earth orbit provides better temporal sampling than is available with a ground-based telescope. We find no compelling evidence for an unusually slow rotation period of multiple days, as has been suggested previously. A $\sim 1.08$ day rotation period is marginally detected at a modest level of statistical confidence $(\sim 97 \%)$. Analysis of the combination of the Swift data with the ground-based B-band measurements of Rabinowitz et al. (2007) returns the same period ( 1.08 day) at a slightly higher statistical confidence $(\sim 99 \%)$.
\end{abstract}

Key words: KUIPER BELT

\section{Introduction}

The recently discovered Kuiper belt object Eris is $1.05 \pm 0.4 \%$ the size of Pluto (Brown et al., 2006). The near-infrared spectrum of Eris is dominated by methane (Brown et al., 2005), suggesting that its surface, like Pluto, is covered in significant deposits of methane frost. The surface of Pluto is variegated, with regions of low and high albedo (Cruikshank et al., 1997). The heterogeneity of Pluto's surface is revealed in its light curve, which has a large amplitude of 0.33 magnitude (Buie et al., 1997). The V band geometric albedo of Eris ( $85 \pm 7 \%$; Brown et al. (2006)) is approximately equal to the albedo of the brightest patches on Pluto's surface (Stern et al., 1997). This led to the suggestion that the surface of Eris is likely homogeneous and of a composition similar to the brightest patches of Pluto (Brown et al., 2006). 
Pluto's rotation period (6.4 days) is set by tidal interactions with its large moon Charon. Dysnomia, the one known moon of Eris, is far too small to have significantly altered the rotation rate of Eris. Several previous observers have not conclusively identified the rotation period of Eris (Carraro et al., 2006; Lin et al., 2007; Sheppard, 2007; Rabinowitz et al., 2007; Duffard et al., 2008). The goal of the observations reported here was to measure the rotation period of Eris.

\section{Observations}

In December 2006 and January 2007 we acquired a sequence of images with the Ultraviolet/Optical Telescope (UVOT) of the Swift spacecraft. The Swift mission was designed to detect Gamma Ray Bursts (GRB) and rapidly slew to measure their optical afterglow. Accepting the risk that observations may be interrupted to follow an evolving GRB, UVOT is available for non-GRB science. While UVOT is small $(30 \mathrm{~cm})$ compared with many groundbased telescopes, UVOT has two distinct advantages for this work. Being space-based UVOT is not subject to the vagaries of weather or atmospheric transparency and is therefore photometrically much more stable than a ground-based telescope. Additionally, being in low-Earth orbit UVOT can observe throughout each 24 hour day, losing only $\sim 45$ minutes out of every $\sim 90$ minute orbit, while a ground-based telescope is limited by its site to observing Eris for 6-10 hours per day. These are particularly useful advantages when an object has a slow rotation period of one day or longer, such as has been suggested for Eris. Over several weeks we acquired nearly $200 \mathrm{ksec}$ of exposure time on Eris with UVOT in the B filter. Most of these images were acquired in a $2 \times 2$ binning mode with a pixel size of $1^{\prime \prime} .0$. The first three images of Table 1 were acquired with no binning $\left(0^{\prime \prime} \cdot 5 / \mathrm{pixel}\right)$ and were rebinned to $2 \times 2$ for the analysis.

Following the photometric prescription of Li et al. (2006) we measured the magnitude of Eris in each individual frame. We found 220 frames taken between 19 December 2006 and 16 January 2007 were of usable quality. (See Table 1 for a full listing of the observations.) To refine the precision of the frame-to-frame relative photometry we also measured an ensemble of 26 comparison stars, chosen to be between 1 magnitude fainter and 2 magnitudes brighter than Eris and to appear in a minimum of 180 of the 220 frames. Several other stars that met these criteria were eliminated for having obvious photometric periodicities or trends. None of these eliminated stars displayed periodicities near the $\sim 1.1$ day period we find for Eris. The mean full-width at half-maximum (FWHM) of Eris and the 26 comparison stars was $2^{\prime \prime} \cdot 3$. We found the aperture radius for optimum signal-to-noise ratio to be close enough to the 3.0 aperture radius recommended by Li et al. (2006) that we adopted a $3^{\prime \prime} .0$ aperture radius throughout. We determined the magnitude correction 
for each frame using the ensemble photometry algorithm of Honeycutt (1992). With the photometric stability of UVOT these corrections are small, but nonnegligible.

The Sun-Eris-Earth distance varied over the time period of observations and the reported measurements have been scaled to remove this known effect $(<1 \%$ over the time period). The Sun-Eris-Earth phase angle also varied over the time period of observations between 0.535 and 0.582 (see Table 1 ). The phase coefficient for Eris in the B-band is zero within uncertainty $(-0.004 \pm 0.028$ mag $\mathrm{deg}^{-1}$; Rabinowitz et al., 2007), implying $\leq 0.0015 \mathrm{mag}$ of variation in the Swift data due to phase angle variation. Any brightness variation due to phase angle is well below our detection limits, and we have not attempted to scale the data to remove the phase angle effect.

The measured count rate of photons from Eris is low, approximately 0.6 photons $\mathrm{sec}^{-1}$, and the estimated uncertainty in any individual measurement is large, ranging between 0.05 and 0.13 mag with a median and mean of 0.08 mag. This spread of uncertainty per measurement is almost entirely explained by variable exposure times, necessitated by telescope and instrument scheduling issues. The resulting measurements of Eris are given in Table 1 and shown in Fig. 1. Also shown in Fig. 1 are the mean daily measured magnitudes of Eris. Weighting the individual measurements by their estimated uncertainties we find a mean B magnitude of Eris of $19.494 \pm 0.007$, where the final uncertainty is estimated from the standard deviation of the measurements divided by $\sqrt{220}$.

Throughout the observations Eris moved across the background field of stars and galaxies and one possible explanation for any observed variability would be the blending of background objects into the aperture used to measure the flux of Eris. Figure 2 is a combined image of the entire dataset with the positions of Eris during our observations overplotted. To test for possible background source contamination we performed aperture photometry, using the same technique as above, for each position on the combined image where we had observed Eris. The standard deviation of this photometry was 0.0004 photons $\mathrm{sec}^{-1}$ with a maximum excursion from the mean of 0.0006 photons $\mathrm{sec}^{-1}$. Given a mean count rate of $\sim 0.6$ photons $\mathrm{sec}^{-1}$ for Eris, the maximum background source contribution to any observed variation in brightness on Eris is 0.001 mag. This is much smaller than the measured photometric uncertainties and demonstrates that background stars and galaxies did not significantly contaminate the measured Eris fluxes. Dysnomia, the known moon of Eris, is far too faint to have influenced our measurements. 


\section{Discussion}

The Lomb-Scargle periodogram (Press et al., 1992) for these data is shown in Fig. 3A. The significance levels are calculated following the technique suggested by Press et al. (1992), which is to shuffle the data, randomly reassigning observed magnitudes to observation times, and recalculate the periodogram. This operation is performed repeatedly and the peak power in each shuffled periodogram is recorded. The significance levels are determined from $1-f$, where $f$ is the fraction of the samples for which the highest peak is greater than the power level in question. Two peaks (at $\sim 1.1$ and $\sim 15$ days) are at suggestive levels of significance. The data are shown phased to these periods in Fig. 3B and 3C.

Periodograms are powerful tools, but have serious limitations. These data and the $\sim 15$ day peak are a case study in some of the hazards of periodograms if incorrectly interpreted. One should be extremely wary of strong peaks at periods longer than $\sim 1 / 3-1 / 2$ of the total time period covered. The phased data of Fig. 3B show significant gaps in data coverage, which is an additional warning sign in periodogram analysis that results may not be trustworthy. From Fig. 3A it would be very tempting to conclude that we detected a 15 day period in Eris, but the data incompletely span only approximately twice this length of time. Examination of the statistics of the variability of the daily mean does not support the detection of a 15 day period. Although the human eye is drawn to the three consecutive daily mean magnitudes in Fig. 1 that lie $>1 \sigma$ fainter than the overall mean of the dataset (JD 2454100-2454102), these appear to be a statistical fluke. A simple Monte Carlo simulation reveals that in a Gaussian noise dataset of 23 points (the number of daily means in Fig. 1) about $36 \%$ of the time there will be three consecutive data points that all sit $1-3 \sigma$ above the mean or all sit $1-3 \sigma$ below the mean. Due to these issues we strongly discount the significance of the peak at $\sim 15$ days.

More interesting is the peak at $\sim 1.1$ days, which has a significance level of 97\%. There are no obvious issues in the phased data of Fig. 3C. To probe the validity of this possible $\sim 1.1$ day period we ran a variety of tests on our data. We split the dataset in half and found a similar result from each half, although with the expected lower confidence level due to fewer data. We ran a sequence of tests in which we randomly selected half of the data points to analyze and again found similar results. We searched the data for any possible correlations, e.g. flux of Eris with position on the detector, but identified none that could explain the signal seen in Fig. 3C or that could not be ruled out by examination of the ensemble of comparison stars. We examined the LombScargle periodogram for each of the 26 comparison stars. As expected, given that we had eliminated potential comparison stars with obvious periodicities, none of the 26 comparison stars displayed peaks in the periodogram above a 
significance level of $90 \%$.

To further test the validity of the possible $\sim 1.1$ day period we combined the Swift dataset with the B-band measurements of Rabinowitz et al. (2007), which is the one other published dataset with a significant number of highquality B-band measurements. After scaling the Swift measurements to the reduced magnitude system of Rabinowitz et al. (2007) the periodogram of the combined dataset is shown in Fig. 4A. The peak of the periodogram remains at approximately the same period (1.08 days) as with the Swift dataset alone, although the significance level of the peak increases slightly to nearly $99 \%$. The combined data phased to this 1.08 day period are shown in Fig. 4B. The results from the combined dataset look very similar to the results from the Swift dataset alone.

We can find no reason to discount the validity of the $\sim 1.1$ day period. Using the $50 \%$ confidence levels of the periodogram as a guide for estimating the uncertainty in the period, we report that Eris appears to be rotating once every $1.08 \pm 0.02$ days with a peak-to-valley amplitude of nearly $0.1 \mathrm{mag}$. At the modest level of confidence available from these data the periodic signal appears less like a sinusoid and more like what would be expected from a large dark patch on the partially hidden hemisphere of an otherwise homogeneous body. At rotational phases where the patch is hidden from the observer the light curve is constant. A dip in the light curve is observed during the rotational phases that the dark patch is visible to the observer. Additional observations will be required to confirm this result and more precisely determine the rotation period of Eris.

Of the previously published datasets the long-term sequence of Rabinowitz et al. (2007) and the several nights of precision photometry of Sheppard (2007) are the most likely to have been able to identify the apparent periodicity seen in these Swift data.

Sheppard (2007) did precision $R$-band photometry of Eris over several hours on each of three contiguous nights in October 2005 and three contiguous nights in December 2005. A careful evaluation of the approximately daily sampling of their data reveals that a periodic signal such as that suggested by the Swift data in Fig. 3C could have been missed. The signal in Fig. 3C appears nearly constant over $\sim 50 \%$ of the rotation period, while phased to a period of 1.08 days the Sheppard (2007) data cover less than half of this rotation period.

The magnitude of variation in the light curve of Eris is likely a function of wavelength. Most of the surface of Eris must be uniformly bright to achieve the high $V$-band geometric albedo (86\% $\pm 7 \%$; Brown et al., 2006). Any darker patches are likely to be photochemically processed hydrocarbons, which will have a red color. At longer wavelengths (e.g. $R$-band and $I$-band) the albedo of 
these red patches will be closer to the albedo of the uniformly bright dominant surface material. At shorter wavelengths (e.g. the B-band used here by Swift) there will be greater contrast between these red patches and the rest of the surface of Eris. This is directly analogous to what is seen on Pluto, where the light curve at $B$-band has an amplitude of $\sim 0.1$ mag greater than the amplitude at $R$-band (Buratti et al., 2003). Thus, the light curve of Eris is likely to be less pronounced at longer wavelengths (e.g. R-band) than the shorter wavelength B-band observed with Swift.

Rabinowitz et al. (2007) reported observations in several filters, taken once per night over many months. The analysis of the Rabinowitz et al. (2007) data is thus dependent upon the simultaneous fitting of color terms and phase function coefficients. We experimented with inserting fake signals into the Rabinowitz et al. (2007) data with a range of periods and amplitudes consistent with the periodicity detected in the Swift data. We then used the same analysis tools as before to search for periodicity. In many cases the inserted periodicity is recovered, however whether the fake signal is detected and the period at which it is detected is strongly dependent on the exact amplitude and period of the fake signal. This is primarily because the test periods $(\sim 1.08$ days) are near the sampling interval ( $\sim 1$ day) of the Rabinowitz et al. (2007) data. In some example cases varying the inserted signal's period by only 0.005 days made the difference between a strong detection and a non-detection. However, as described above, the combination of the B-band measurements of Rabinowitz et al. (2007) with the Swift measurements reported here improves the significance of the retrieved periodicity somewhat from that of the Swift data alone.

A coherent picture of Eris is emerging. The surface is primarily covered in bright methane frost, much like the brightest patches of Pluto's surface. However, our results suggest that the surface of Eris is not perfectly homogeneous. Under thermal equilibrium the vapor pressure of methane on Eris is negligible at its current near-perihelion distance of $97.5 \mathrm{AU}$. If the entire surface of Eris is uniformly covered in very high albedo methane frost, even at aphelion (38.2 AU) the equilibrium surface temperature of Eris is not warm enough to generate significant methane evaporation from the surface. This presents a problem as methane frost in the outer solar system is expected to redden and darken due to photochemical processing, but Eris appears bright and shows no sign of redness. This suggests the methane frost deposits on its surface must be fresh and a replenishment mechanism is required. Our detection of variability is consistent with regional darker areas on the surface. At aphelion the widespread high albedo regions will not warm enough to sublimate methane into the atmosphere, however the small darker areas will be heated by the increased insolation to kickoff feedback effects that lead to dramatic global surface change, generating a temporary atmosphere and replenishing the methane surface each Eris year. 


\section{Acknowledgements}

We thank David Rabinowitz and an anonymous referee.

\section{References}

Brown, M. E., Schaller, E. L., Roe, H. G., Rabinowitz, D. L., Trujillo, C. A., May 2006. Direct Measurement of the Size of 2003 UB313 from the Hubble Space Telescope. Astrophysical Journal Letters 643, L61-L63.

Brown, M. E., Trujillo, C. A., Rabinowitz, D. L., Dec. 2005. Discovery of a Planetary-sized Object in the Scattered Kuiper Belt. Astrophysical Journal Letters 635, L97-L100.

Buie, M. W., Tholen, D. J., Wasserman, L. H., Feb. 1997. Separate Lightcurves of Pluto and Charon. Icarus 125, 233-244.

Buratti, B. J., Hillier, J. K., Heinze, A., Hicks, M. D., Tryka, K. A., Mosher, J. A., Ward, J., Garske, M., Young, J., Atienza-Rosel, J., Mar. 2003. Photometry of pluto in the last decade and before: evidence for volatile transport? Icarus 162, 171-182.

Carraro, G., Maris, M., Bertin, D., Parisi, M. G., Dec. 2006. Time series photometry of the dwarf planet ERIS (2003 UB313). Astronomy \& Astrophysics 460, L39-L42.

Cruikshank, D. P., Roush, T. L., Moore, J. M., Sykes, M., Owen, T. C., Bartholomew, M. J., Brown, R. H., Tryka, K. A., 1997. The Surfaces of Pluto and Charon. Pluto and Charon, pp. 221-+.

Duffard, R., Ortiz, J. L., Santos Sanz, P., Mora, A., Gutiérrez, P. J., Morales, N., Guirado, D., Mar. 2008. A study of photometric variations on the dwarf planet (136199) Eris. Astronomy \& Astrophysics 479, 877-881.

Honeycutt, R. K., Jun. 1992. CCD ensemble photometry on an inhomogeneous set of exposures. Publications of the Astronomical Society of the Pacific 104, 435-440.

Li, W., Jha, S., Filippenko, A. V., Bloom, J. S., Pooley, D., Foley, R. J., Perley, D. A., Jan. 2006. The Calibration of the Swift UVOT Optical Observations: A Recipe for Photometry. Publications of the Astronomical Society of the Pacific 118, 37-61.

Lin, H.-W., Wu, Y.-L., Ip, W.-H., 2007. Observations of dwarf planet (136199) Eris and other large TNOs on Lulin Observatory. Advances in Space Research 40, 238-243.

Press, W. H., Teukolsky, S. A., Vetterling, W. T., Flannery, B. P., 1992. Numerical recipes in FORTRAN. The art of scientific computing. Cambridge: University Press, - c1992, 2nd ed.

Rabinowitz, D. L., Schaefer, B. E., Tourtellotte, S. W., Jan. 2007. The Diverse Solar Phase Curves of Distant Icy Bodies. I. Photometric Observations of 
18 Trans-Neptunian Objects, 7 Centaurs, and Nereid. Astronomical Journal $133,26-43$.

Sheppard, S. S., Aug. 2007. Light Curves of Dwarf Plutonian Planets and other Large Kuiper Belt Objects: Their Rotations, Phase Functions, and Absolute Magnitudes. Astronomical Journal 134, 787-798.

Stern, S. A., Buie, M. W., Trafton, L. M., Feb. 1997. HST High-Resolution Images and Maps of Pluto. Astronomical Journal 113, 827-885. 


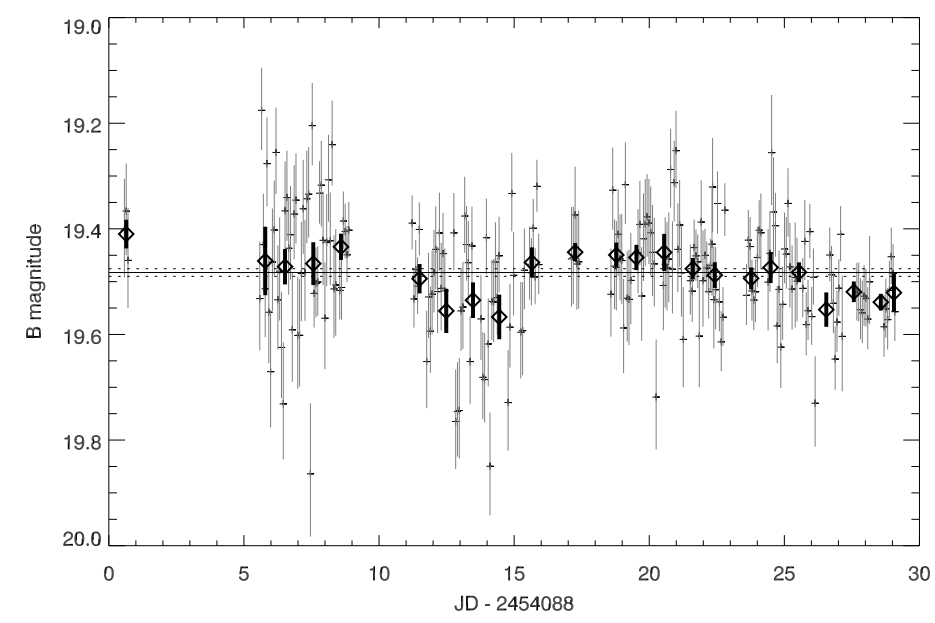

Fig. 1. Each of the 220 Swift measurements of Eris in the $B$ filter is shown along with its estimated photometric uncertainty. Overplotted as diamonds are the daily means of the data with uncertainties estimated from the standard deviation within each day. Also shown is the mean magnitude of the entire dataset, with $1 \sigma$ estimated uncertainty.

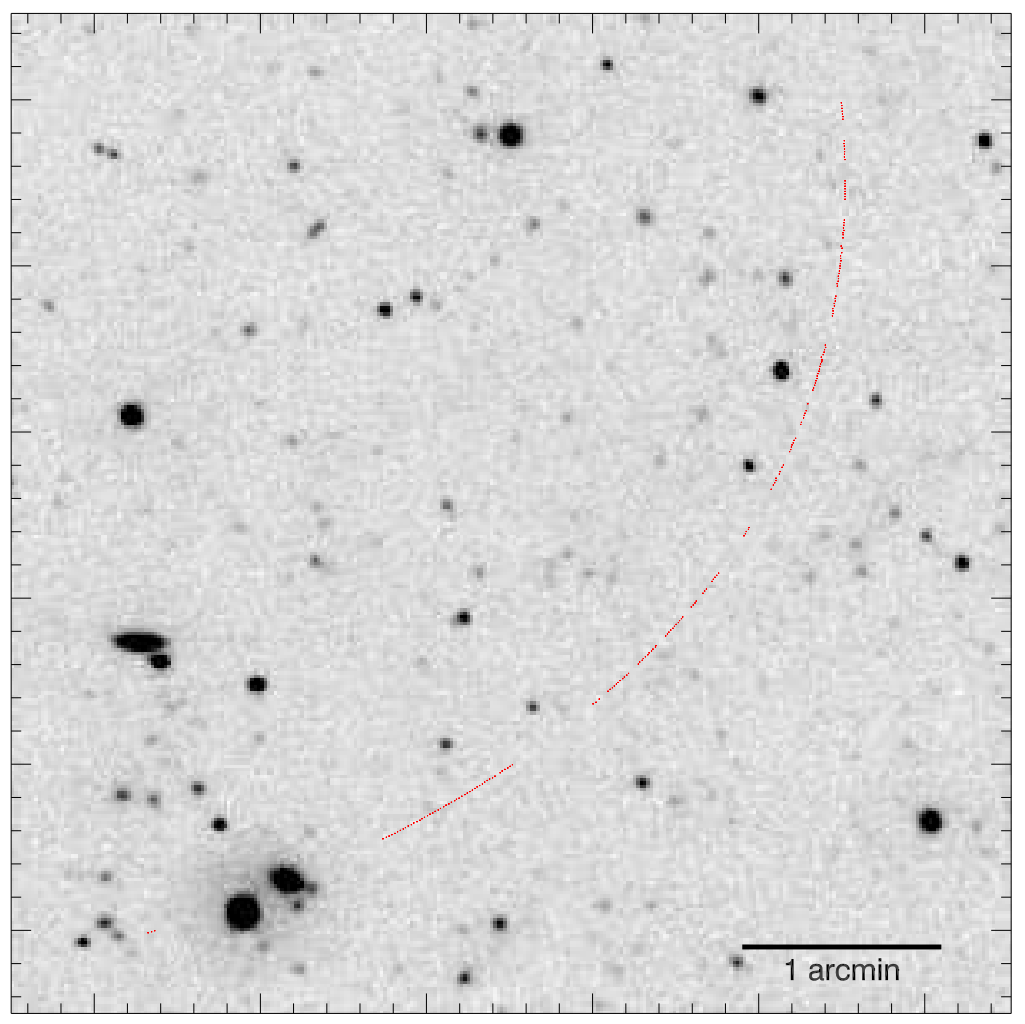

Fig. 2. Combination of all Swift B images. The gray scale of the image is scaled linearly between $\pm 0.3 \%$ of the mean flux of Eris. Overplotted is the position of Eris at the time of each exposure used in the analysis. 

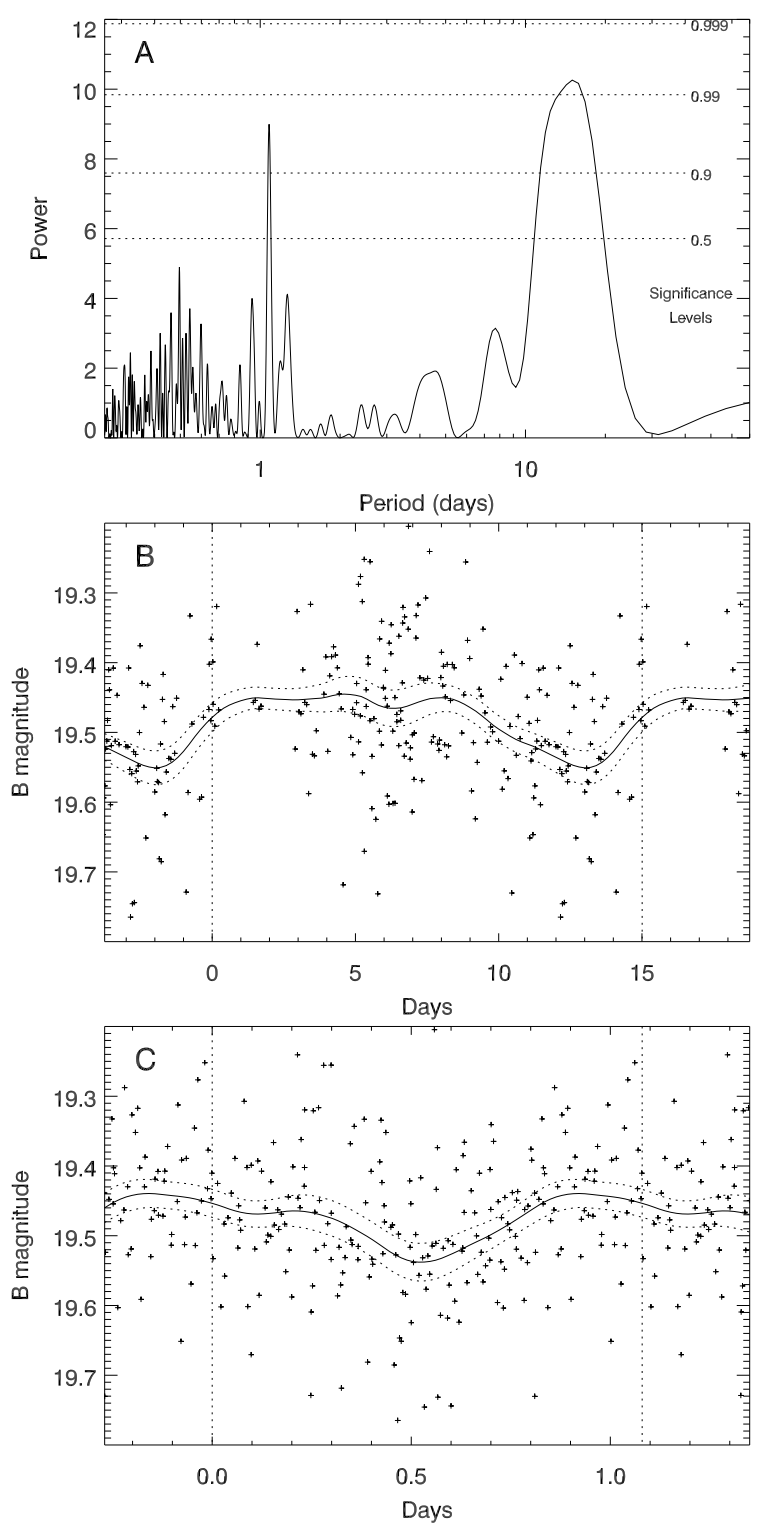

Fig. 3. (A) Periodogram of Swift data calculated using the FASPER algorithm of Press et al. (1992). The significance levels are calculated via a Monte Carlo approach as suggested in Press et al. (1992). (B) Swift measurements of Eris phased to a period of 15.0 days. Continuously overplotted is a running mean of the nearest 20 phased data points, along with the $1 \sigma$ estimated uncertainty in that mean. (C)Swift measurements of Eris phased to a period of 1.08 days. Continuously overplotted is a running mean of the nearest 20 phased data points, along with the $1 \sigma$ estimated uncertainty in that mean. We strongly discount the calculated significance level of the 15 day period as our dataset covered only 28 days. 

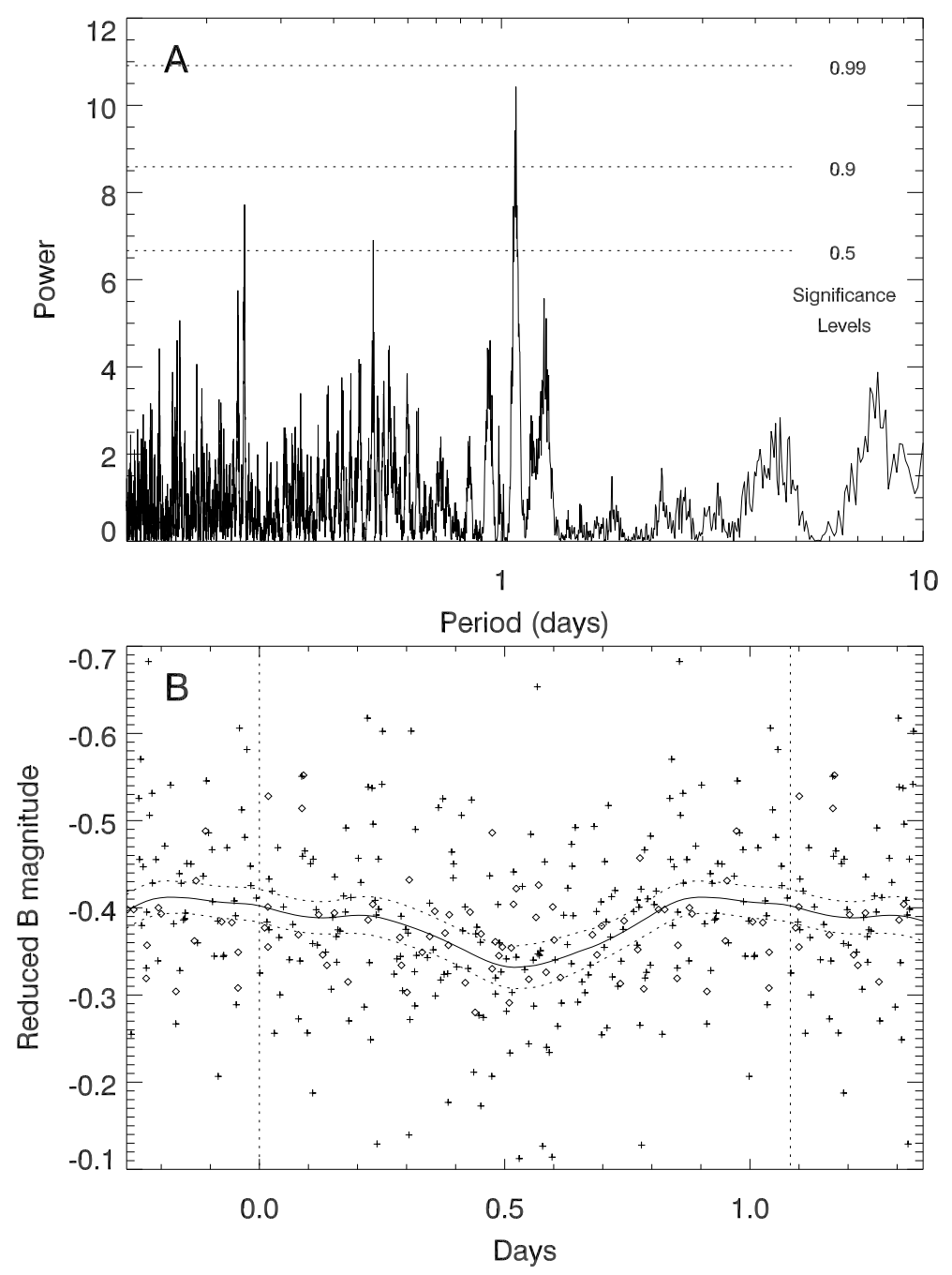

Fig. 4. (A) Periodogram of the combined Swift and Rabinowitz et al. (2007) dataset calculated using the FASPER algorithm of Press et al. (1992). The significance levels are calculated via a Monte Carlo approach as suggested in Press et al. (1992). (C) Combined dataset phased to a period of 1.08 days. Swift data are shown as plus signs (+), while Rabinowitz et al. (2007) data are shown as diamonds $(\diamond)$. Continuously overplotted is a running mean of the nearest 20 phased data points, along with the $1 \sigma$ estimated uncertainty in that mean. The addition of the Rabinowitz et al. (2007) data slightly improve the statistical significance of the recovered periodicity. 
Table 1: Table 1: Swift measurements.

\begin{tabular}{|c|c|c|c|}
\hline Julian Date & Exp. time (s) & B Magnitude & Phase angle \\
\hline 2454088.576 & 390.5 & $19.40 \pm 0.09$ & 0.535 \\
\hline 2454088.643 & 390.5 & $19.37 \pm 0.09$ & 0.536 \\
\hline 2454088.710 & 391.0 & $19.46 \pm 0.09$ & 0.536 \\
\hline 2454093.585 & 450.4 & $19.53 \pm 0.10$ & 0.553 \\
\hline 2454093.652 & 450.9 & $19.18 \pm 0.08$ & 0.553 \\
\hline 2454093.719 & 450.8 & $19.43 \pm 0.09$ & 0.553 \\
\hline 2454093.786 & 450.9 & $19.51 \pm 0.10$ & 0.553 \\
\hline 2454093.853 & 450.5 & $19.28 \pm 0.08$ & 0.554 \\
\hline 2454093.920 & 450.4 & $19.56 \pm 0.10$ & 0.554 \\
\hline 2454093.987 & 450.9 & $19.67 \pm 0.11$ & 0.554 \\
\hline 2454094.054 & 451.0 & $19.46 \pm 0.10$ & 0.554 \\
\hline 2454094.121 & 451.0 & $19.40 \pm 0.09$ & 0.554 \\
\hline 2454094.188 & 451.0 & $19.26 \pm 0.08$ & 0.555 \\
\hline 2454094.255 & 451.5 & $19.53 \pm 0.10$ & 0.555 \\
\hline 2454094.322 & 450.8 & $19.48 \pm 0.10$ & 0.555 \\
\hline 2454094.389 & 450.9 & $19.62 \pm 0.10$ & 0.555 \\
\hline 2454094.456 & 450.4 & $19.73 \pm 0.11$ & 0.555 \\
\hline 2454094.522 & 450.9 & $19.37 \pm 0.09$ & 0.556 \\
\hline 2454094.590 & 451.0 & $19.34 \pm 0.09$ & 0.556 \\
\hline 2454094.656 & 450.9 & $19.44 \pm 0.09$ & 0.556 \\
\hline 2454094.724 & 450.9 & $19.41 \pm 0.09$ & 0.556 \\
\hline 2454094.790 & 450.4 & $19.59 \pm 0.10$ & 0.557 \\
\hline 2454094.857 & 450.4 & $19.37 \pm 0.09$ & 0.557 \\
\hline 2454094.924 & 450.4 & $19.35 \pm 0.09$ & 0.557 \\
\hline 2454094.991 & 449.9 & $19.60 \pm 0.11$ & 0.557 \\
\hline 2454095.058 & 449.4 & $19.60 \pm 0.10$ & 0.557 \\
\hline 2454095.125 & 450.8 & $19.48 \pm 0.09$ & 0.557 \\
\hline
\end{tabular}




\begin{tabular}{|c|c|c|c|}
\hline 2454095.192 & 450.9 & $19.36 \pm 0.09$ & 0.558 \\
\hline 2454095.259 & 450.4 & $19.48 \pm 0.10$ & 0.558 \\
\hline 2454095.326 & 450.5 & $19.34 \pm 0.09$ & 0.558 \\
\hline 2454095.393 & 450.5 & $19.33 \pm 0.09$ & 0.558 \\
\hline 2454095.460 & 450.5 & $19.86 \pm 0.13$ & 0.558 \\
\hline 2454095.527 & 450.4 & $19.20 \pm 0.08$ & 0.559 \\
\hline 2454095.597 & 922.9 & $19.52 \pm 0.07$ & 0.559 \\
\hline 2454095.664 & 863.9 & $19.50 \pm 0.07$ & 0.559 \\
\hline 2454095.731 & 921.9 & $19.50 \pm 0.07$ & 0.559 \\
\hline 2454095.797 & 923.4 & $19.33 \pm 0.06$ & 0.559 \\
\hline 2454095.862 & 449.0 & $19.32 \pm 0.08$ & 0.559 \\
\hline 2454095.929 & 450.0 & $19.42 \pm 0.09$ & 0.560 \\
\hline 2454095.996 & 450.1 & $19.57 \pm 0.10$ & 0.560 \\
\hline 2454096.063 & 450.5 & $19.43 \pm 0.09$ & 0.560 \\
\hline 2454096.129 & 450.5 & $19.31 \pm 0.08$ & 0.560 \\
\hline 2454096.197 & 450.5 & $19.42 \pm 0.09$ & 0.560 \\
\hline 2454096.263 & 451.0 & $19.24 \pm 0.08$ & 0.561 \\
\hline 2454096.331 & 450.5 & $19.51 \pm 0.10$ & 0.561 \\
\hline 2454096.397 & 450.4 & $19.51 \pm 0.10$ & 0.561 \\
\hline 2454096.546 & 1337.1 & $19.52 \pm 0.06$ & 0.561 \\
\hline 2454096.611 & 982.2 & $19.51 \pm 0.06$ & 0.561 \\
\hline 2454096.679 & 1159.4 & $19.39 \pm 0.05$ & 0.562 \\
\hline 2454096.747 & 1336.2 & $19.41 \pm 0.05$ & 0.562 \\
\hline 2454096.814 & 1336.2 & $19.45 \pm 0.05$ & 0.562 \\
\hline 2454096.881 & 1336.6 & $19.40 \pm 0.05$ & 0.562 \\
\hline 2454099.224 & 1311.0 & $19.39 \pm 0.05$ & 0.568 \\
\hline 2454099.291 & 1370.8 & $19.53 \pm 0.06$ & 0.568 \\
\hline 2454099.358 & 1312.3 & $19.48 \pm 0.05$ & 0.568 \\
\hline 2454099.425 & 1371.5 & $19.51 \pm 0.05$ & \\
\hline
\end{tabular}




$\begin{array}{llll}2454099.492 & 1372.5 & 19.40 \pm 0.05 & 0.568 \\ 2454099.764 & 574.3 & 19.65 \pm 0.09 & 0.569 \\ 2454099.831 & 632.7 & 19.53 \pm 0.08 & 0.569 \\ 2454099.898 & 632.8 & 19.59 \pm 0.08 & 0.569 \\ 2454099.965 & 632.7 & 19.52 \pm 0.08 & 0.569 \\ 2454100.032 & 632.7 & 19.48 \pm 0.08 & 0.569 \\ 2454100.099 & 633.3 & 19.44 \pm 0.08 & 0.569 \\ 2454100.166 & 632.6 & 19.52 \pm 0.08 & 0.570 \\ 2454100.233 & 632.7 & 19.41 \pm 0.07 & 0.570 \\ 2454100.300 & 632.7 & 19.51 \pm 0.08 & 0.570 \\ 2454100.366 & 632.1 & 19.45 \pm 0.07 & 0.570 \\ 2454100.434 & 632.0 & 19.52 \pm 0.08 & 0.570 \\ 2454100.768 & 634.6 & 19.41 \pm 0.07 & 0.571 \\ 2454100.835 & 634.5 & 19.76 \pm 0.09 & 0.571 \\ 2454100.902 & 693.0 & 19.75 \pm 0.09 & 0.571 \\ 2454100.969 & 634.6 & 19.74 \pm 0.10 & 0.571 \\ 2454101.036 & 693.0 & 19.56 \pm 0.08 & 0.571 \\ 2454101.103 & 634.5 & 19.55 \pm 0.08 & 0.571 \\ 2454101.170 & 634.5 & 19.38 \pm 0.07 & 0.572 \\ 2454101.237 & 693.5 & 19.43 \pm 0.07 & 0.572 \\ 2454101.304 & 634.7 & 19.46 \pm 0.08 & 0.572 \\ 2454101.371 & 693.1 & 19.65 \pm 0.08 & 0.572 \\ 2454101.438 & 693.1 & 19.43 \pm 0.07 & 0.572 \\ 2454101.772 & 634.2 & 19.57 \pm 0.08 & 0.573 \\ 2454101.840 & 693.5 & 19.68 \pm 0.08 & 0.573 \\ 2454101.906 & 693.7 & 19.69 \pm 0.08 & 0.573 \\ 2454101.973 & 693.2 & 19.42 \pm 0.07 & 0.573 \\ 2454102.040 & 693.5 & 19.62 \pm 0.08 & 0.573 \\ 2452.107 & 693.7 & 19.85 \pm 0.10 & 0.573\end{array}$




$\begin{array}{llll}2454102.174 & 693.0 & 19.54 \pm 0.08 & 0.573 \\ 2454102.241 & 693.2 & 19.54 \pm 0.08 & 0.573 \\ 2454102.308 & 693.4 & 19.46 \pm 0.07 & 0.574 \\ 2454102.375 & 693.6 & 19.53 \pm 0.07 & 0.574 \\ 2454102.442 & 693.5 & 19.45 \pm 0.07 & 0.574 \\ 2454102.777 & 575.2 & 19.73 \pm 0.10 & 0.574 \\ 2454102.844 & 634.1 & 19.59 \pm 0.08 & 0.574 \\ 2454102.911 & 574.6 & 19.33 \pm 0.07 & 0.574 \\ 2454102.978 & 634.2 & 19.49 \pm 0.08 & 0.575 \\ 2454103.246 & 575.2 & 19.60 \pm 0.09 & 0.575 \\ 2454103.313 & 634.2 & 19.59 \pm 0.09 & 0.575 \\ 2454103.380 & 634.2 & 19.48 \pm 0.08 & 0.575 \\ 2454103.571 & 664.4 & 19.47 \pm 0.07 & 0.575 \\ 2454103.639 & 900.2 & 19.48 \pm 0.06 & 0.576 \\ 2454103.708 & 1195.2 & 19.40 \pm 0.05 & 0.576 \\ 2454103.776 & 1431.9 & 19.49 \pm 0.05 & 0.576 \\ 2454103.842 & 1196.2 & 19.32 \pm 0.05 & 0.576 \\ 2454103.909 & 1254.8 & 19.47 \pm 0.06 & 0.576 \\ 2454105.121 & 403.3 & 19.46 \pm 0.09 & 0.577 \\ 2454105.188 & 439.0 & 19.45 \pm 0.09 & 0.578 \\ 2454105.255 & 416.2 & 19.37 \pm 0.09 & 0.578 \\ 2454105.322 & 452.0 & 19.47 \pm 0.09 & 0.578 \\ 2454105.389 & 428.7 & 19.46 \pm 0.09 & 0.578 \\ 2454106.582 & 545.9 & 19.52 \pm 0.08 & 0.579 \\ 2454106.648 & 486.1 & 19.33 \pm 0.08 & 0.579 \\ 2454106.715 & 486.6 & 19.47 \pm 0.09 & 0.579 \\ 2454106.782 & 487.5 & 19.47 \pm 0.08 & 0.579 \\ 2454106.849 & 487.1 & 19.41 \pm 0.08 & 0.579 \\ 2456.916 & 487.1 & 19.46 \pm 0.08 & 0.579\end{array}$




\begin{tabular}{|c|c|c|c|}
\hline 2454106.983 & 487.1 & $19.46 \pm 0.09$ & 0.579 \\
\hline 2454107.049 & 486.5 & $19.59 \pm 0.09$ & 0.579 \\
\hline 2454107.117 & 487.0 & $19.32 \pm 0.08$ & 0.580 \\
\hline 2454107.183 & 487.0 & $19.53 \pm 0.09$ & 0.580 \\
\hline 2454107.251 & 486.5 & $19.53 \pm 0.09$ & 0.580 \\
\hline 2454107.317 & 486.7 & $19.50 \pm 0.09$ & 0.580 \\
\hline 2454107.585 & 487.1 & $19.45 \pm 0.08$ & 0.580 \\
\hline 2454107.652 & 487.2 & $19.39 \pm 0.08$ & 0.580 \\
\hline 2454107.719 & 487.1 & $19.53 \pm 0.09$ & 0.580 \\
\hline 2454107.786 & 487.1 & $19.42 \pm 0.08$ & 0.580 \\
\hline 2454107.853 & 487.2 & $19.39 \pm 0.08$ & 0.580 \\
\hline 2454107.919 & 487.2 & $19.38 \pm 0.08$ & 0.580 \\
\hline 2454107.987 & 487.0 & $19.39 \pm 0.08$ & 0.580 \\
\hline 2454108.053 & 487.0 & $19.41 \pm 0.08$ & 0.580 \\
\hline 2454108.121 & 487.7 & $19.44 \pm 0.09$ & 0.580 \\
\hline 2454108.187 & 487.1 & $19.47 \pm 0.08$ & 0.580 \\
\hline 2454108.254 & 487.6 & $19.72 \pm 0.10$ & 0.580 \\
\hline 2454108.522 & 487.0 & $19.51 \pm 0.09$ & 0.580 \\
\hline 2454108.589 & 486.6 & $19.47 \pm 0.09$ & 0.581 \\
\hline 2454108.656 & 486.1 & $19.47 \pm 0.08$ & 0.581 \\
\hline 2454108.723 & 486.6 & $19.46 \pm 0.09$ & 0.581 \\
\hline 2454108.789 & 487.5 & $19.29 \pm 0.07$ & 0.581 \\
\hline 2454108.857 & 487.0 & $19.48 \pm 0.09$ & 0.581 \\
\hline 2454108.924 & 487.1 & $19.31 \pm 0.08$ & 0.581 \\
\hline 2454108.991 & 487.0 & $19.25 \pm 0.07$ & 0.581 \\
\hline 2454109.058 & 486.6 & $19.44 \pm 0.08$ & 0.581 \\
\hline 2454109.124 & 487.6 & $19.39 \pm 0.08$ & 0.581 \\
\hline 2454109.192 & 487.0 & $19.48 \pm 0.09$ & 0.581 \\
\hline 2454109.258 & 487.0 & $19.61 \pm 0.09$ & 0.581 \\
\hline
\end{tabular}




$\begin{array}{llll}2454109.521 & 1089.7 & 19.50 \pm 0.07 & 0.581 \\ 2454109.590 & 1355.8 & 19.52 \pm 0.06 & 0.581 \\ 2454109.660 & 1571.7 & 19.44 \pm 0.05 & 0.581 \\ 2454109.728 & 1748.0 & 19.45 \pm 0.05 & 0.581 \\ 2454109.795 & 1749.5 & 19.46 \pm 0.05 & 0.581 \\ 2454109.853 & 617.8 & 19.60 \pm 0.10 & 0.581 \\ 2454109.921 & 671.8 & 19.39 \pm 0.08 & 0.581 \\ 2454109.988 & 553.6 & 19.50 \pm 0.09 & 0.581 \\ 2454110.063 & 1749.6 & 19.45 \pm 0.05 & 0.581 \\ 2454110.129 & 1750.0 & 19.47 \pm 0.05 & 0.581 \\ 2454110.197 & 1749.4 & 19.52 \pm 0.05 & 0.581 \\ 2454110.263 & 1749.3 & 19.47 \pm 0.05 & 0.581 \\ 2454110.321 & 488.2 & 19.43 \pm 0.10 & 0.581 \\ 2454110.344 & 384.0 & 19.32 \pm 0.09 & 0.581 \\ 2454110.388 & 665.2 & 19.53 \pm 0.10 & 0.581 \\ 2454110.457 & 901.4 & 19.52 \pm 0.08 & 0.581 \\ 2454110.526 & 1043.6 & 19.35 \pm 0.06 & 0.581 \\ 2454110.595 & 1515.9 & 19.54 \pm 0.06 & 0.581 \\ 2454110.663 & 1575.4 & 19.61 \pm 0.06 & 0.581 \\ 2454110.730 & 1575.7 & 19.57 \pm 0.06 & 0.581 \\ 2454110.796 & 1575.9 & 19.36 \pm 0.05 & 0.581 \\ 2454111.598 & 1452.3 & 19.53 \pm 0.06 & 0.581 \\ 2454111.666 & 1452.3 & 19.42 \pm 0.06 & 0.582 \\ 2454111.732 & 1452.4 & 19.43 \pm 0.05 & 0.582 \\ 2454111.799 & 1451.9 & 19.52 \pm 0.06 & 0.582 \\ 2454111.867 & 1363.2 & 19.53 \pm 0.06 & 0.582 \\ 2454111.934 & 1364.2 & 19.52 \pm 0.06 & 0.582 \\ 2454112.001 & 1364.1 & 19.45 \pm 0.06 & 0.582 \\ 24112.068 & 1364.2 & 19.40 \pm 0.06 & 0.582\end{array}$




\begin{tabular}{|c|c|c|c|}
\hline 2454112.131 & 753.5 & $19.41 \pm 0.07$ & 0.582 \\
\hline 2454112.397 & 546.4 & $19.50 \pm 0.10$ & 0.582 \\
\hline 2454112.465 & 841.1 & $19.45 \pm 0.08$ & 0.582 \\
\hline 2454112.530 & 389.5 & $19.26 \pm 0.10$ & 0.582 \\
\hline 2454112.597 & 449.0 & $19.37 \pm 0.10$ & 0.581 \\
\hline 2454112.671 & 980.2 & $19.39 \pm 0.06$ & 0.581 \\
\hline 2454112.735 & 980.2 & $19.58 \pm 0.07$ & 0.581 \\
\hline 2454112.802 & 929.6 & $19.51 \pm 0.07$ & 0.581 \\
\hline 2454112.870 & 921.2 & $19.62 \pm 0.08$ & 0.581 \\
\hline 2454112.936 & 921.4 & $19.54 \pm 0.07$ & 0.581 \\
\hline 2454113.004 & 921.2 & $19.44 \pm 0.07$ & 0.581 \\
\hline 2454113.070 & 920.8 & $19.45 \pm 0.07$ & 0.581 \\
\hline 2454113.137 & 921.3 & $19.35 \pm 0.06$ & 0.581 \\
\hline 2454113.206 & 597.1 & $19.47 \pm 0.08$ & 0.581 \\
\hline 2454113.287 & 613.2 & $19.51 \pm 0.08$ & 0.581 \\
\hline 2454113.401 & 512.4 & $19.49 \pm 0.10$ & 0.581 \\
\hline 2454113.470 & 808.0 & $19.50 \pm 0.08$ & 0.581 \\
\hline 2454113.681 & 1438.5 & $19.51 \pm 0.06$ & 0.581 \\
\hline 2454113.756 & 611.5 & $19.42 \pm 0.08$ & 0.581 \\
\hline 2454113.809 & 1599.0 & $19.58 \pm 0.06$ & 0.581 \\
\hline 2454113.876 & 1599.1 & $19.56 \pm 0.06$ & 0.581 \\
\hline 2454113.943 & 1598.8 & $19.41 \pm 0.05$ & 0.581 \\
\hline 2454114.010 & 1598.5 & $19.57 \pm 0.06$ & 0.581 \\
\hline 2454114.077 & 1598.0 & $19.49 \pm 0.05$ & 0.581 \\
\hline 2454114.140 & 1008.6 & $19.73 \pm 0.09$ & 0.581 \\
\hline 2454114.679 & 1639.6 & $19.45 \pm 0.05$ & 0.581 \\
\hline 2454114.746 & 1577.3 & $19.49 \pm 0.05$ & 0.581 \\
\hline 2454114.813 & 1576.9 & $19.54 \pm 0.06$ & 0.581 \\
\hline 2454114.880 & 1577.4 & $19.65 \pm 0.06$ & 0.581 \\
\hline
\end{tabular}




\begin{tabular}{lccl}
2454114.947 & 1577.3 & $19.58 \pm 0.06$ & 0.581 \\
2454115.014 & 1577.3 & $19.51 \pm 0.05$ & 0.581 \\
2454115.081 & 1576.9 & $19.41 \pm 0.05$ & 0.581 \\
2454115.141 & 565.6 & $19.60 \pm 0.11$ & 0.581 \\
2454115.683 & 1629.4 & $19.52 \pm 0.05$ & 0.580 \\
2454115.750 & 1630.0 & $19.52 \pm 0.06$ & 0.580 \\
2454115.818 & 1629.1 & $19.55 \pm 0.06$ & 0.580 \\
2454115.884 & 1591.1 & $19.56 \pm 0.06$ & 0.580 \\
2454115.951 & 1591.9 & $19.53 \pm 0.06$ & 0.580 \\
2454116.018 & 1595.2 & $19.53 \pm 0.06$ & 0.580 \\
2454116.085 & 1595.0 & $19.57 \pm 0.06$ & 0.580 \\
2454116.154 & 560.4 & $19.50 \pm 0.08$ & 0.580 \\
2454116.688 & 1594.7 & $19.59 \pm 0.06$ & 0.580 \\
2454116.755 & 1594.2 & $19.55 \pm 0.06$ & 0.580 \\
2454116.822 & 1594.2 & $19.57 \pm 0.06$ & 0.580 \\
2454116.889 & 1594.0 & $19.52 \pm 0.05$ & 0.580 \\
2454116.955 & 1594.6 & $19.45 \pm 0.05$ & 0.580 \\
2454117.023 & 1593.2 & $19.48 \pm 0.05$ & 0.580 \\
2454117.089 & 1594.6 & $19.56 \pm 0.06$ & 0.579 \\
\hline
\end{tabular}

